\title{
van der Waals Interactions between Thin Metallic Wires and Layers
}

\author{
N. D. Drummond and R. J. Needs \\ TCM Group, Cavendish Laboratory, University of Cambridge, Cambridge CB3 OHE, United Kingdom
} (Received 30 May 2007; published 16 October 2007)

\begin{abstract}
Quantum Monte Carlo (QMC) methods have been used to obtain accurate binding-energy data for pairs of parallel thin metallic wires and layers modeled by 1D and 2D homogeneous electron gases. We compare our QMC binding energies with results obtained within the random phase approximation, finding significant quantitative differences and disagreement over the asymptotic behavior for bilayers at low densities. We have calculated pair-correlation functions for metallic biwire and bilayer systems. Our QMC data could be used to investigate van der Waals energy functionals.
\end{abstract}

One-dimensional conductors such as carbon nanotubes are essential components of many proposed nanotechnological devices, and they are currently the subject of numerous experimental and theoretical studies. It has recently been demonstrated [1] that the van der Waals ( $\mathrm{vdW})$ interaction between pairs of distant parallel thin conducting wires assumed in many current models of metallic carbon nanotubes is qualitatively wrong. In this Letter we provide the first accurate binding-energy data for pairs of thin metallic wires and layers, which can be used as a benchmark for subsequent theoretical studies or to parametrize model interactions between $1 \mathrm{D}$ and $2 \mathrm{D}$ conductors.

Thin electrically neutral wires are attracted to one another by vdW forces. The standard method for calculating the vdW interaction between objects is to assume there are pairwise interactions between volume elements with an attractive tail of the form $U_{\mathrm{PP}}(r) \propto-r^{-6}$, which is appropriate for the vdW interaction between molecules. Summing these interactions for a pair of 1D parallel wires separated by a distance $z$ gives a vdW binding energy of $U(z) \propto-z^{-5}$. Such pairwise vdW models have been used in studies of single-walled carbon nanotubes [2,3]. However, a recent investigation [1] of the interaction between pairs of thin, metallic wires modeled by $1 \mathrm{D}$ homogeneous electron gases (HEGs) within the random phase approximation (RPA) found that the binding energy falls off (approximately) as [4]

$$
U(z) \approx-\frac{\sqrt{r_{s}}}{16 \pi z^{2}[\log (2.39 z / b)]^{3 / 2}},
$$

where $b$ is the wire radius and $2 r_{s}$ is the length of the wire section containing one electron on average. The pairwise $\mathrm{vdW}$ model is clearly appropriate for an insulator or for a metallic wire whose radius is greater than the screening length but is inappropriate for a thin conductor such as a single-walled carbon nanotube [1].

Likewise, the binding energy per particle of a pair of thin parallel metallic layers can be shown to decay as

$$
U(z)=\frac{-0.012562 \sqrt{\pi} r_{s}}{2 z^{5 / 2}}
$$

within the RPA [1,5], compared with $U(z) \propto-z^{-4}$ within the pairwise $\mathrm{vdW}$ theory, where $z$ is the layer separation. (In a 2D HEG $r_{s}$ is the radius of the circle that contains one electron on average.) At very large separations the vdW attraction is dominated by the Casimir effect $[5,6]$, in which the zero-point energy of photon modes between the metallic layers gives rise to an attractive force. However, we restrict our attention to the range of separations in which vdW effects are dominant.

Within the RPA, the binding energy may be calculated as the change in the zero-point energy of plasmon modes as a function of separation [1]. However, the RPA is poor in low-dimensional systems and ceases to be valid at low densities, where correlation effects become dominant. We have therefore performed quantum Monte Carlo [7,8] (QMC) calculations of the binding energies of pairs of thin, metallic wires and layers modeled by $1 \mathrm{D}$ and 2D HEGs with neutralizing backgrounds. In particular, we have used the variational and diffusion quantum Monte Carlo (VMC and DMC) methods as implemented in the CASINO code [9]. DMC is the most accurate method available for studying quantum many-body systems such as electron gases. We have also calculated pair-correlation functions (PCFs), enabling us to examine the correlation hole responsible for the vdW attraction between pairs of wires and layers.

In our QMC calculations we use the full Coulomb potential, so that for 1D HEGs the many-electron wave function must go to zero at both parallel- and antiparallelspin coalescence points for electrons in the same wire. The nodal surfaces for paramagnetic and ferromagnetic 1D HEGs are therefore the same, and so the fixed-node DMC energy - which is equal to the exact ground-state energy because the nodal surface is exact-is independent of the spin polarization. This conclusion does not violate the Lieb-Mattis theorem [10] because the 1D Coulomb interaction is pathological in the formal sense of Lieb and Mattis [11]. For convenience, we choose to work 
with ferromagnetic 1D HEGs, because a Slater determinant wave function produces the correct nodal surface in this case. Our QMC studies of 1D HEGs will be published elsewhere [12].

Fermionic symmetry is imposed in $2 \mathrm{D}$ via the fixednode approximation [13], in which the nodal surface is constrained to equal that of a trial wave function. We expect a very high degree of cancellation of fixed-node errors when the binding energy is calculated. It has already been shown that fixed-node DMC is able to describe vdW forces between helium [14] and neon atoms [15].

We have verified that time-step and population-control biases in our DMC energies are negligible by repeating some of the calculations using different time steps and populations. Finite-size errors are a more serious problem, although most of the bias cancels out when the energy difference is taken to obtain the binding energy. Twist averaging [16] or the addition of finite-size corrections are unlikely to reduce the bias in the binding energy. We expect our binding-energy results to be valid so long as the wire or layer separation is small compared with the length of the 1D or 2D simulation cell; for larger separations the system resembles a pair of insulators and the binding energy is expected to fall off more steeply (in accordance with the pairwise vdW model).

We have used Slater-Jastrow-backflow trial wave functions $[8,17]$. For our 2D bilayer calculations the Slater part of the wave function consists of a product of four determinants of plane-wave orbitals for spin-up and spin-down electrons in each of the two layers. For our 1D biwire calculations the Slater part consists of a product of two determinants of plane-wave orbitals for the electrons in each wire (recall that each wire is ferromagnetic in our calculations). Slater determinants for a 1D HEG are of Vandermonde form and could therefore be rewritten as polynomials and evaluated in a time that scales linearly with system size; however, other parts of the QMC algorithm such as the evaluation of the two-body Jastrow terms and backflow functions take up a significant fraction of the computer time, so for convenience we have continued to employ the usual determinant-evaluating and updating machinery of QMC calculations [18].

Our Jastrow factors consist of polynomial and planewave two-body terms [19] satisfying the Kato cusp conditions [20]. In spite of the fact that the nodal surface is exact in 1D, two-body backflow correlations [17] were found to make a very significant improvement to the wave function, as can be seen in Table I. Backflow functions were used in all of our 1D calculations and in our 2D calculations at $r_{s}=1$ a.u.. Free parameters in the trial wave function were optimized by minimizing the unreweighted variance of the local energy [21,22].

Each wire or layer is accompanied by a neutralizing background. When studying biwires (bilayers) we need to add the interaction between the electrons in each wire (layer) and the background of the opposite wire (layer), plus the interaction of the two backgrounds. This contribution to the energy is $E_{\text {cap }}=\log (z) /\left(2 r_{s}\right)$ for a biwire and $E_{\text {cap }}=z / r_{s}^{2}$ for a bilayer.

The binding energies of pairs of 1D and 2D HEGs are shown in Figs. 1 and 2. The approximate RPA binding energy shown in Fig. 1 was obtained using $b=r_{s} / 10$ in Eq. (1). The wire radius $b$ is therefore small compared with the other length scales in the system. We have included DMC results at several different system sizes in Figs. 1 and 2. In 1D, half the length of the simulation cell is $L_{r_{s}, N}=$ $N r_{s}$, where $N$ is the number of electrons per wire, and in 2D the size of the simulation cell is $L_{r_{s}, N} \approx \sqrt{N} r_{s}$. The binding energy falls off more steeply once $z$ becomes a significant fraction of $L_{r_{s}, N}$, as expected. Clearly the binding energies enter the asymptotic regime when $z \gg r_{s}$. We have therefore fitted the RPA asymptotic binding-energy forms to our QMC data in the range $r_{s} \ll z \ll L_{r_{s}, N}$. We believe the errors in the fitted exponents are about 0.1-0.2.

The fits to the DMC biwire binding-energy data shown in Fig. 1 are

$$
\begin{gathered}
U_{1}(z)=-0.0815 z^{-2.28}[\log (27000 z)]^{-3 / 2} \\
U_{3}(z)=-0.0225 z^{-1.98}[\log (1.95 z)]^{-3 / 2} \\
U_{10}(z)=-0.0967 z^{-2.17}[\log (0.492 z)]^{-3 / 2},
\end{gathered}
$$

where $U_{r_{s}}(z)$ is the binding energy at density parameter $r_{s}$. The DMC binding-energy data are clearly in much better agreement with the RPA [Eq. (1)] than with the pairwise vdW theory $\left[U(z) \propto z^{-5}\right]$. It is not meaningful to compare the prefactors because of the arbitrariness of our choice of the wire radius $b$ in the RPA theory.

The fits to the DMC bilayer binding-energy data shown in Fig. 2 are

TABLE I. Energy, energy variance, and fraction of correlation energy retrieved using different levels of theory and wave function for a 15-electron 1D ferromagnetic HEG at $r_{s}=15$ a.u. "HF" stands for Hartree-Fock theory, "SJ" denotes a Slater-Jastrow trial wave function, and "SJB" a Slater-Jastrow-backflow trial wave function.

\begin{tabular}{llcc}
\hline \hline Method & Energy (a.u. per electron) & Variance (a.u.) & Percentage correlation energy \\
\hline HF & -0.215943040112 & $\ldots$ & $0 \%$ \\
SJ-VMC & $-0.2319668(4)$ & $0.0000360(2)$ & $99.974(3) \%$ \\
SJB-VMC & $-0.2319710(3)$ & $0.0000046(1)$ & $100.000(3) \%$ \\
SJB-DMC & $-0.2319709(3)$ & $\ldots$ & $100 \%$ \\
\hline \hline
\end{tabular}



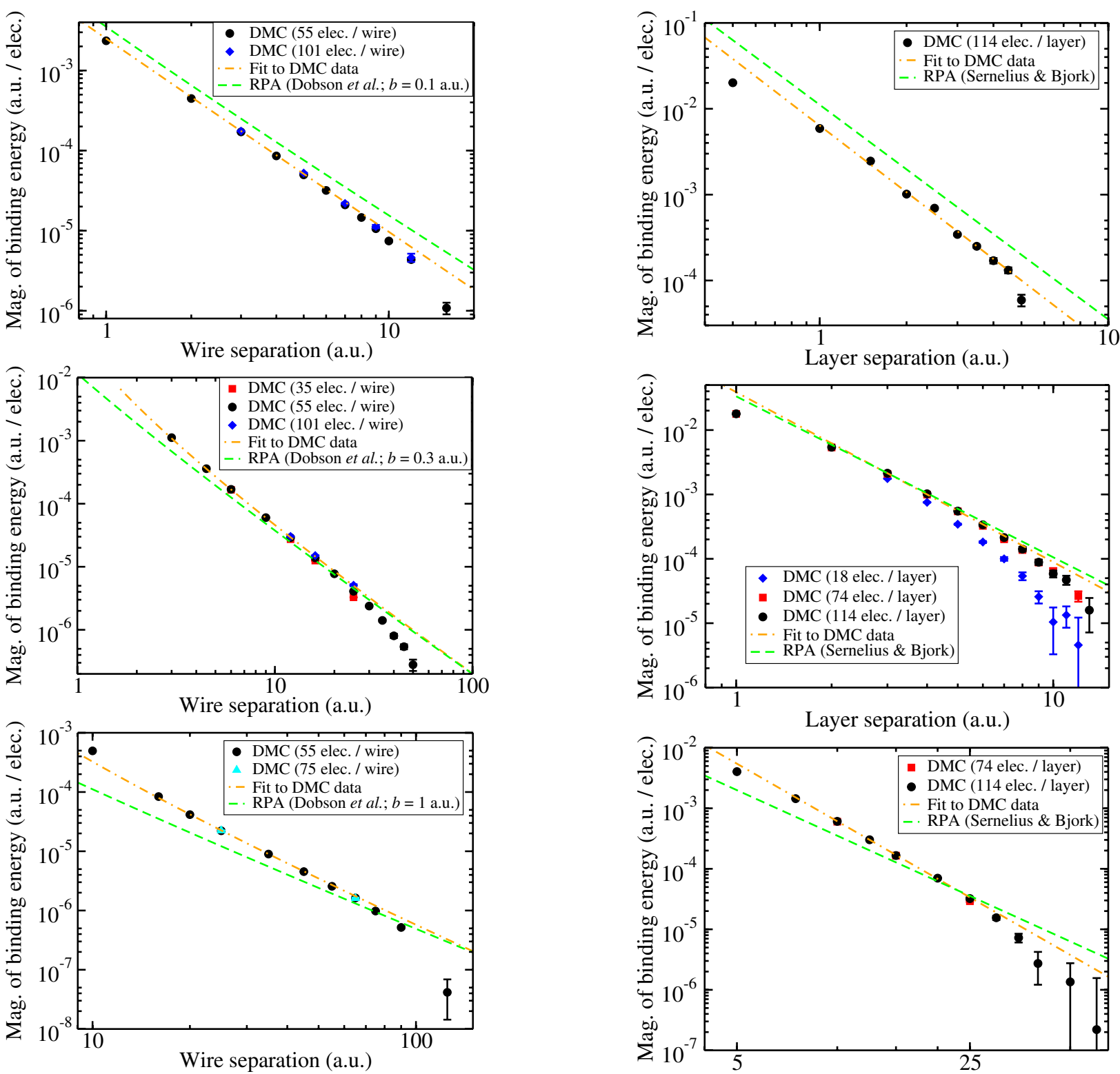

FIG. 1 (color online). Binding energy per particle of a 1D HEG biwire as a function of wire separation for $r_{s}=1$ a.u. (top panel), $r_{s}=3$ a.u. (middle panel), and $r_{s}=10$ a.u. (bottom panel). The DMC time steps were 0.04, 0.2, and 2.5 a.u. at $r_{s}=$ 1,3 , and 10 a.u., and the target configuration population was 2048 in each case.

$$
\begin{aligned}
& U_{1}(z)=-0.00637 z^{-2.58} \\
& U_{3}(z)=-0.0388 z^{-2.64} \\
& U_{10}(z)=-0.882 z^{-3.16},
\end{aligned}
$$

where $U_{r_{s}}(z)$ is the binding energy at density parameter $r_{s}$. At high densities ( $r_{s}=1$ and 3 a.u.) our results clearly show the $-z^{-2.5}$ behavior predicted by Eq. (2). At low density ( $r_{s}=10$ a.u.) the binding energy falls off more steeply than predicted by the RPA, although the asymptotic behavior is clearly better described by the RPA than the

FIG. 2 (color online). Binding energy per particle of a $2 \mathrm{D}$ HEG bilayer as a function of layer separation for $r_{s}=1$ a.u. (top panel), $r_{s}=3$ a.u. (middle panel), and $r_{s}=10$ a.u. (bottom panel). The DMC time steps were $0.007,0.05$, and 0.5 a.u. at $r_{s}=1,3$, and 10 a.u., and the target configuration populations were 1024,320 , and 1024 .

pairwise vdW theory $\left[U(z) \propto-z^{-4}\right]$. DMC and the RPA give similar prefactors for the asymptotic binding energy at $r_{s}=3$ a.u., but the DMC prefactor is somewhat lower at $r_{s}=1$ a.u.

PCFs were accumulated by binning the interparticle distances in the electron configurations generated by the VMC and DMC algorithms. The error in the VMC and DMC PCFs $g_{\mathrm{VMC}}$ and $g_{\mathrm{DMC}}$ is first order in the error in the trial wave function, but the error in the extrapolated PCF $g_{\text {ext }}=2 g_{\text {DMC }}-g_{\text {VMC }}$ is second order in the error in the wave function [8]. PCFs for biwires and bilayers are shown 


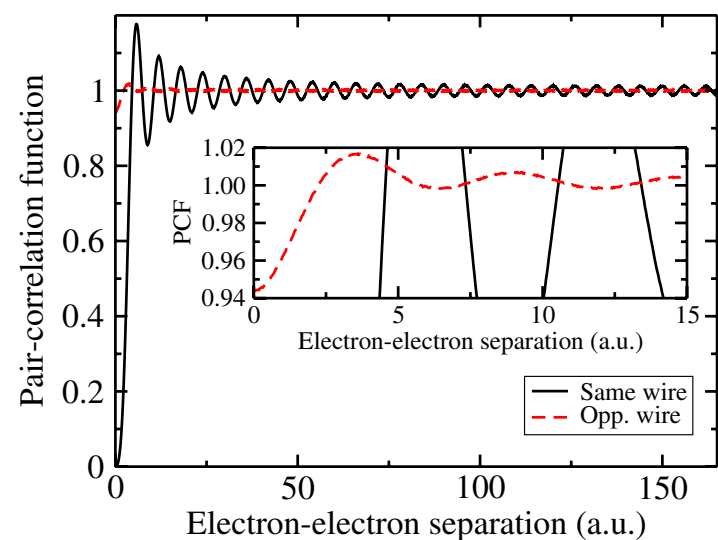

FIG. 3 (color online). VMC PCF for a 1D HEG biwire at $r_{s}=$ 3 a.u. and wire separation $z=3$ a.u. The inset shows the interwire correlation hole in greater detail. It was verified for smaller system sizes that the DMC and VMC PCFs were in excellent agreement (as expected due to the accuracy of the trial wave function illustrated in Table I).

in Figs. 3 and 4, respectively. The correlation holes between the electrons in opposite wires or planes are exceedingly shallow, although they extend over a distance roughly equal to the separation. It can be seen that the interwire PCF has the long-ranged oscillatory behavior exhibited by 1D HEG PCFs.

In conclusion, we have used QMC to obtain the first accurate binding-energy data for pairs of thin parallel metallic wires and layers. Our results are in broad agreement with recent RPA calculations of the binding energy and complete disagreement with the standard pairwise $\mathrm{vdW}$ model. However, there are significant differences between the DMC and RPA results for bilayers: at high densities the asymptotic behavior of the binding energy as a function of separation is the same but the prefactor is different, and at low densities the DMC binding energy falls off more rapidly, implying that correlation effects

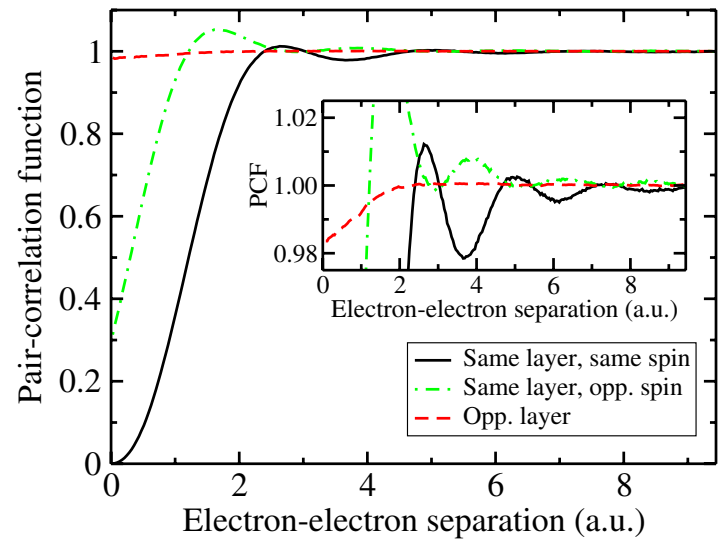

FIG. 4 (color online). Extrapolated PCF for a 2D HEG bilayer at $r_{s}=1$ a.u. and layer separation $z=2$ a.u. The inset shows the interwire correlation hole in greater detail. The VMC and DMC PCFs are in excellent agreement, implying that the extrapolated PCF is reliable. neglected in the RPA are important in this regime. Our data can serve as a benchmark for future theoretical studies of the binding energies of 1D and 2D HEGs, and they can also be used to parametrize model interactions between thin conductors. Together with our results for biwire and bilayer PCFs, our data could be used to investigate energy functionals that incorporate vdW effects for use in densityfunctional calculations.

We acknowledge financial support from Jesus College, Cambridge and the UK Engineering and Physical Sciences Research Council. Computing resources were provided by the Cambridge High Performance Computing Service.

[1] J. F. Dobson, A. White, and A. Rubio, Phys. Rev. Lett. 96, 073201 (2006).

[2] L. A. Girifalco, M. Hodak, and R. S. Lee, Phys. Rev. B 62, 13104 (2000).

[3] C.-H. Sun, L.-C. Yin, F. Li, G.-Q. Lu, and H.-M. Cheng, Chem. Phys. Lett. 403, 343 (2005).

[4] We use Hartree atomic units $\left(\hbar=|e|=m_{e}=4 \pi \epsilon_{0}=1\right)$ throughout this Letter.

[5] B. E. Sernelius and P. Björk, Phys. Rev. B 57, 6592 (1998).

[6] H. B. G. Casimir and D. Polder, Phys. Rev. 73, 360 (1948).

[7] D. M. Ceperley and B. J. Alder, Phys. Rev. Lett. 45, 566 (1980).

[8] W. M. C. Foulkes, L. Mitas, R. J. Needs, and G. Rajagopal, Rev. Mod. Phys. 73, 33 (2001).

[9] R. J. Needs, M. D. Towler, N. D. Drummond, and P. López Ríos, CASINO version 2.1 User Manual (University of Cambridge, Cambridge, England, 2007).

[10] E. Lieb and D. Mattis, Phys. Rev. 125, 164 (1962).

[11] For pathological 1D interactions, which force the wave function to be 0 at points other than same-spin coalescences, the weak form of the Lieb-Mattis theorem implies that the energy of the paramagnetic phase is less than or equal to that of the ferromagnetic phase; this is consistent with our conclusion that the paramagnetic and ferromagnetic phases are degenerate in energy.

[12] N. D. Drummond and R. J. Needs (to be published).

[13] J. B. Anderson, J. Chem. Phys. 65, 4121 (1976).

[14] J.B. Anderson, C.A. Traynor, and B. M. Boghosian, J. Chem. Phys. 99, 345 (1993); J. B. Anderson, J. Chem. Phys. 115, 4546 (2001).

[15] N.D. Drummond and R. J. Needs, Phys. Rev. B 73, 024107 (2006).

[16] C. Lin, F. H. Zong, and D. M. Ceperley, Phys. Rev. E 64, 016702 (2001).

[17] P. López Ríos, A. Ma, N. D. Drummond, M. D. Towler, and R. J. Needs, Phys. Rev. E 74, 066701 (2006).

[18] S. Fahy, X. W. Wang, and S. G. Louie, Phys. Rev. B 42, 3503 (1990).

[19] N. D. Drummond, M. D. Towler, and R. J. Needs, Phys. Rev. B 70, 235119 (2004).

[20] T. Kato, Commun. Pure Appl. Math. 10, 151 (1957); R. T. Pack and W. B. Brown, J. Chem. Phys. 45, 556 (1966).

[21] C. J. Umrigar, K. G. Wilson, and J. W. Wilkins, Phys. Rev. Lett. 60, 1719 (1988).

[22] N.D. Drummond and R. J. Needs, Phys. Rev. B 72, 085124 (2005). 